3 Orfanos SE, Langleben D, Khoury J, et al. Pulmonary capillary endothelium-bound angiotensin-converting enzyme activity in humans. Circulation 1999; 99: 1593-1599.

4 Orfanos SE, Psevdi E, Stratigis N, et al. Pulmonary capillary endothelial dysfunction in early systemic sclerosis. Arthritis Rheum 2001; 44: 902-911.
5 Orfanos SE, Hirsch AM, Giovinazzo M, et al. Pulmonary capillary endothelial metabolic function in chronic thromboembolic pulmonary hypertension. J Thromb Haemost 2008; 6: 1275-1280.

DOI: 10.1183/09031936.00130409

\title{
Nonthrombotic pulmonary embolism
}

\section{To the Editors:}

We read with great interest the article in the European Respiratory Journal by JORENS et al. [1] on the different types of nonthrombotic pulmonary embolism. Among them, hydatid disease is of major importance as it is endemic in sheep-raising regions worldwide. We wish, therefore, to outline the management of metastatic pulmonary hydatidosis secondary to a primary hydatid cyst located in the liver according to our experience of six cases.

First, the diagnosis of hydatid pulmonary vascular obstruction should be looked for systematically in patients with a primary hepatic hydatid cyst located near the inferior vena cava and/or supra-hepatic veins, by perfusion lung scan and/or spiral thoracic computed tomography.

Secondly, once the pulmonary vascular obstruction has been diagnosed, the surgical treatment of the hepatic cyst at the origin of the embolism must be carried out as the first step. However, mobilisation of the liver may cause hydatid embolism and haemorrhagic complications when the hydatid cysts are in contact with the walls of the inferior vena cava. We suggest the following preventive measures: a wide laparotomy to control the inferior vena cava and have an extracorporeal bypass ready. These guidelines may prevent the fatal intraoperative pulmonary embolism as previously reported, and which occurred in two patients from our series [2].

Thirdly, surgery for a hydatid pulmonary vascular obstruction is quite similar to that for usual pulmonary embolism: embolectomy by arteriotomy for proximal pulmonary intra vascular hydatid cysts (five times in four patients in our series) using cardiopulmonary bypass in two cases.

Finally, chronic pulmonary arterial hypertension may worsen even after hydatid embolectomy as the vascular obstruction is also distal and associated with a granulomatous reaction and vascular fibrosis [3]. Pulmonary transplantation may be a therapeutic option because immunosuppressive treatment does not adversely affect the course of hydatid disease [4]. Recently, the use of pulmonary hypertension medical therapy, such as endothelin-1-receptor antagonists, phosphodiesterase5 -inhibitors and prostacyclin analogues, has been reported to be clinically effective [5].

\section{Vinatier*, E. Rivaud*, C. Tcherakian*, A. Bisson", B. Goudot ${ }^{\top}$ and L.J. Couderc* \\ *Depts of Pneumonology, "Thoracic Surgery, and "Cardio- vascular Surgery, Hopital Foch, Suresnes, France.}

Correspondence: L.J. Couderc, Service de Pneumologie, Hôpital Foch, 40 rue Worth, 92150 Suresnes, France. E-mail: lj.couderc@hopital-foch.org

Statement of Interest: None declared.

\section{REFERENCES}

1 Jorens PG, Van Marck E, Snoeckx A, et al. Nonthrombotic pulmonary embolism. Eur Respir J 2009; 34: 452-474.

2 Rothlin MA. Fatal intraoperative pulmonary embolism from a hepatic hydatid cyst. Am J Gastroenterol 1998; 93: 2606-2607.

3 Deve F. Ecchinococcose pulmonaire metastatique. In: Ecchinococcose secondaire. Paris, Masson, 1946; pp. 145-178.

4 Sobrino JM, Pulpon LA, Crespo MG, et al. Heart transplantation in a patient with liver hydatidosis. J Heart Lung Transplant 1993; 12: 531-533.

5 Bulman W, Coyle CM, Brentjens TE, et al. Severe pulmonary hypertension due to chronic echinococcal pulmonary emboli treated with targeted pulmonary vascular therapy and hepatic resection. Chest 2007; 132: 1356-1358.

\section{Effects of nanoparticles on lung damage in humans}

\section{To the Editors:}

We read with great interest a recent article in the European Respiratory Journal by SoNG et al. [1] and we would like to add some questions/comments to their paper. First, we are interested in the (estimated) total weight of the polyacrylate nanoparticles detected in the lungs and/or pleural fluids obtained from the patients. In our in vivo experiments, mice were exposed seven times per week to nanoparticles (carbon black [2] and latex [3]) at the weight of $50 \mu \mathrm{g} \cdot \mathrm{time}^{-1} \cdot \mathrm{animal}^{-1}$ ( $\sim 83 \mathrm{mg}$ in the human body), and did not die within 6 months thereafter (unpublished observation). Taken together, results 
from the study in humans by SONG et al. 11] and our in vivo studies may provide an important finding regarding difference in susceptibility to nanoparticles between humans and rodents. The second point to be addressed is the possibility of endotoxin attaching to (around) the nanoparticles. Do the authors have any information about endotoxin level in the lung samples or endotoxin concentration in the workplace? We have previously demonstrated that combined exposure to nanoparticles and endotoxin elicited devastating lung injury compared to nanoparticles or endotoxin alone [4]. The lethal events occurring in the patients may have resulted from synergistic effects of nanoparticles with other toxic substances, rather than effects of the nanoparticles alone. Finally, we would like to know if these seven patients had pre-existing atopy. In particular, we are interested in the physical condition of the patients described in "The general characteristics of the patients" section in which "all patients suffered from rash with intense itching on their faces" [1], implicating an association with some immunological impairment. Allergen-specific immunoglobulin (Ig)E titres examined by radioallergosorbent test could provide hints for detection, as total IgE (measured by radioimmunosorbent test) does not always cover allergic conditions. Furthermore, atopic subjects are prone to particulate matter (PM), i.e. PM exposure significantly exacerbates the pathophysiology in the subjects $[2,5]$, sometimes leading to a fatal outcome. A careful search for pre-existing illness (in particular, atopy) may have helped in resolving the pathogenesis of the lung damage seen in the patients. In any case, the study by SONG et al. [1] is valuable for future inhalation toxicology, and environmental and preventive medicine, if correlated to previous in vivo findings.

\section{K. Inoue and H. Takano}

National Institute for Environmental Studies, Tsukuba, Japan.

Correspondence: K. Inoue, Environmental Health Sciences Division, National Institute for Environmental Studies, 16-2 Onogawa, Tsukuba, 305-8506, Japan. E-mail: inoue.kenichirou @nies.go.jp

Statement of Interest: None declared.

\section{REFERENCES}

1 Song Y, Li X, Du X. Exposure to nanoparticles is related to pleural effusion, pulmonary fibrosis and granuloma. Eur Respir J 2009; 34: 559-567.

2 Inoue K, Takano H, Yanagisawa R, et al. Effects of nano particles on antigen-related airway inflammation in mice. Respir Res 2005; 6: 106.

3 Inoue K, Takano H, Yanagisawa R, et al. Size effects of latex nanomaterials on lung inflammation in mice. Toxicol Appl Pharmacol 2009; 234: 68-76.

4 Inoue K, Takano H, Yanagisawa R, et al. Effects of airway exposure to nanoparticles on lung inflammation induced by bacterial endotoxin in mice. Environ Health Perspect 2006; 114: 1325-1330.

5 Dockery DW, Pope CA 3rd, Xu X, et al. An association between air pollution and mortality in six U.S. cities. N Engl J Med 1993; 329: $1753-1759$.

DOI: $10.1183 / 09031936.00135609$

\section{From the authors:}

We would like to thank $\mathrm{K}$. Inoue and $\mathrm{H}$. Takano for their comments and questions regarding our paper entitled "Exposure to nanoparticles is related to pleural effusion, pulmonary fibrosis and granuloma" [1].

We read with great interest the study mentioned by $\mathrm{K}$. Inoue and H. Takano [2] with the knowledge that combined exposure to nanoparticles and endotoxin elicited devastating lung injury in comparison to nanoparticles or endotoxin alone. However, with regard to the patients in our study, no evidence was found to show that their symptoms might be related to endotoxin from their occupational exposure, our on-the-spot investigation, clinical observations and examinations, or longterm follow-up. If the patients had inhaled the endotoxin, some symptoms and signs of "endotoxin poisoning" would exist, such as fever, tiredness, headache or hypotension, but none of these were observed.

In general, two mechanisms are involved in the toxicity of nanoparticles. One mechanism is that nanoparticles themselves directly exert toxicities, which are related to the chemical component, size and shape of nanoparticles [3]. When some nanomaterials gain entry into the body, either via inhalation, dermal or oral routes, and penetrate into cells, they can subsequently pose a series of cytotoxicities or promote DNA damage by several mechanisms [4]. For example, nanoparticles can physically interact with the DNA molecule or proteins, which may lead to physical damage to the cell or genetic material. In addition, inflammation and oxidative stress (generation of reactive oxygen species) induced by nanoparticles have been identified as giving rise to effects on cell membranes, cytoplasm, nuclei and mitochondrial function $[4,5]$. Importantly, nanoparticles can damage cells through the regulation of redox-sensitive transcription factors, induction of apoptotic and necrotic cell death and decreased proliferation, and DNA damage responsive signalling [4-6]. A recent study has shown that cationic starburst polyamidoamine dendrimer (PAMAM) nanoparticles trigger autophagic cell death by deregulating the Akt-TSC2-mTOR signalling pathway, and induce acute lung injury in vivo [7]. The other mechanism of nanoparticle toxicity is that nanoparticles can be used as delivery carriers [8]. In cancer and gene therapy, nanoparticles can deliver drugs at high concentrations to the sites of interest, e.g. cancer lesions. It is not difficult to understand that if the material the nanoparticles carry is not a drug but highly toxicant, it may cause potential damage to cells.

In our study, "nanoparticles were observed to lodge in the cytoplasm and caryoplasm of pulmonary epithelial and mesothelial cells" [1], direct interaction between nanoparticles and the DNA molecule or DNA-related proteins may lead to physical damage to the genetic material. In addition, nanoparticles may exert toxicities on cells by other mechanisms, such as inflammation, oxidative stress or cell responsive signalling. In order to question whether the lethal events resulted from the synergy of nanoparticls with other toxic substances, or to what extent the illnesses were due to particles and other toxic substances, further animal experiments need to be performed in order to draw a firm conclusion. However, in our opinion, as the workers were used the exposure of powder coatings, as well as other reasons 